internalizing problems (Health-related 0.08, 0.04-0.12; Poverty/discord 0.08, 0.01-0.16; Multiple 0.24, 0.15-0.34). Adding childcare and interactions with adversity yielded negative interaction terms for High Centre $\mathrm{x}$ Poverty/discord on the externalizing intercept $(-1.83,-3.18$ to -0.47$)$; and for High Combined $\mathrm{x}$ Poverty/discord on the externalizing intercept ($1.70,-3.23$ to -0.15$)$, internalizing intercept $(-1.52,-2.44$ to $0.60)$, and internalizing linear slope $(-0.23,-0.44$ to -0.02$)$. Corresponding interaction terms for Health-related adversity were generally small and negative, but for Multiple adversity were larger and all positive: for both these adversity subtypes, confidence intervals spanned zero. Sensitivity analyses incorporating teacher- and child-reported 122/152-month outcomes confirmed main findings.

Conclusion Centre-based care, especially when combined with individual non-parental care, buffered children's mental health from family adversity characterised by poverty and interparental discord. Limitations include low statistical power for small subgroups and lack of childcare quality information. Findings indicate childcare may be protective against some, but not all, patterns of family adversity.

\section{P45 EXERCISING TO CONTROL SIGNS AND SYMPTOMS OF STRESS AND DEPRESSION}

\begin{abstract}
1,2JA Martin*, 1,2,3 CE Gheorge, ${ }^{4} \mathrm{M}$ Molloy, ${ }^{5} \mathrm{~K}$ O'Halloran, ${ }^{1,2} \mathrm{TG}$ Dinan, ${ }^{1,2,3} \mathrm{JF}$ Cryan, $1,2,6 \mathrm{G}$ Clarke. 'Department of Psychiatry and Neurobehavioural Science, University College Cork, Cork, Ireland; ${ }^{2}$ APC Microbiome Ireland, University College Cork, Cork, Ireland; ${ }^{3}$ Department of Anatomy and Neuroscience, University College Cork, Cork, Ireland; ${ }^{4}$ Clinical Research Ethics Committee of the Cork Teaching Hospitals, University College Cork, Cork, Ireland; ${ }^{5}$ Department of Physiology, University College Cork, Cork, Ireland; ${ }^{6}$ INFANT Research Centre, University College Cork, Cork, Ireland
\end{abstract}

\subsection{6/jech-2020-SSMabstracts. 139}

Background 300 million people worldwide suffer from depression. A poorly understood stress-related pathophysiology is compounded by frequent treatment-resistance.

Undertaking regular exercise has consistently shown to prevent, protect and increase resilience against the development of aberrant stress-related responses seen in depression, whereas sedentary behaviour tends to heighten symptoms. Despite the promise shown by exercise its clinical use is undermined by poor understanding of how beneficial effects are produced.

Stress exposure can dysregulate important physiological pathways such as the hypothalamic-pituitary-adrenal (HPA) axis and the sympathetic nervous system. Disruption of tryptophan metabolism, including increased metabolism along the kynurenine pathway, is a neurobiological hallmark of depressed patients. Recent studies have shown that the beneficial impact of exercise for mood and cognition may be linked to reduced kynurenine production.

The aim was to examine the benefits of a 12 -week exercise programme in healthy sedentary adults across mood, stress, anxiety and depression were mediated by the regulation of tryptophan metabolism in a dose dependent manner.

Methods 32 healthy sedentary adults participated (16 females). To establish a baseline profile each participant undertook a battery of psychometric assessments, cognitive assessments and a cycle ergometer-based incremental fitness assessment. Measures of oxygen uptake and capillary blood lactate levels were taken at the end of each stage during the fitness assessment.
After the baseline assessment, participants were randomised into one of four groups (high, moderate, low dose exercise, or the sedentary control group). Exercise was administered over 3 sessions/week based on individual fitness and group. Monthly fitness and psychometric assessments were performed throughout to monitor change across the 12-week exercise programme. Data analyses were performed using SPSS.

Results As expect, participants showed a positive increase in fitness and performance with an increase in maximal oxygen uptake $(F(1,28)=14.62, p=0.001, \eta p 2=0.343)$ after 12 -weeks exercise training, accompanied by an increased relative peak power output $(\mathrm{rPPO})(\mathrm{F}(1,28)=16.93, \mathrm{p}=0.000, \eta \mathrm{p} 2=0.377)$ and an increased time to exhaustion (T2Ex) $(\mathrm{F}(1,28)=31.07$, $\mathrm{p}=0.000, \eta \mathrm{p} 2=0.526)$. Post hoc paired T-tests revealed that the most pronounced effects on rPPO and T2Ex occurred in the moderate or high intensity groups There was a reduction in scores on the Beck Depression Inventory (BDI) $(\mathrm{F}(1,28)$ $=6.50, \mathrm{p}=0.017, \eta \mathrm{p} 2=0.189)$ and the Perceived Stress Scale (PSS) $(\mathrm{F}(1,28)=4.09, \mathrm{p}=0.053, \eta \mathrm{p} 2=0.127)$ scores after 12 weeks of exercise.

Conclusion In summary, these results confirm that exercise training beneficially influences measures of stress and depression. Further work is required to understand the role of exercise-induced alterations in tryptophan metabolism in mediating these effects.

\section{P46 THE MEANING OF RECOVERY AT THE INTERSECTION OF ETHNICITY AND GENDER FOR AFRICAN AND CARIBBEAN MEN IN ENGLAND}

${ }^{1} \mathrm{~K}$ Southby*, ${ }^{2} \mathrm{~F}$ Keating, ${ }^{2} \mathrm{~S}$ Joseph. ${ }^{1}$ Centre for Health Promotion Research, Leeds Beckett University, Leeds, UK; ${ }^{2}$ Department for Social Work, Royal Holloway, University of London, London, UK

10.1136/jech-2020-SSMabstracts. 140

Background 'Recovery' is a contested concept within mental health. This is to the detriment of patients' progression. The aim of this paper is to consider the meaning of recovery for African and Caribbean men with mental health experience in England.

Methods A qualitative design using a phenomenological approach captured the dynamics of recovery processes and outcomes for African and Caribbean men across the two study sites (Leeds and London). Fifty-nine in-depth semi-structured interviews were conducted with African and Caribbean men with mental health experience $(n=30)$, supporters/family carers $(n=15)$, and service providers $(n=14)$. Data were analysed using Interpretative Phenomenological Analysis (IPA). At two co-creation events, service users co-analysed the data.

Results Findings suggest that recovery for African and Caribbean men is a complex, dynamic concept. A number of interconnected and often overlapping aspects collectively represent and contribute to recovery. These include recovery as a healing journey, leading a 'normal' life, autonomy and control, aspirations for the future, identity, and being free from health services.

Conclusion Whilst previous research similarly highlights issues of autonomy, social inclusion, personalisation, and identity as fundamental to recovery for all service users, this paper argues that recovery for African and Caribbean men is flavoured by their lived-experience at the intersections of ethnicity and gender. Service providers should acknowledge the men's personal and collective understanding of recovery based on their unique life histories. 